OPEN ACCESS

Edited by:

Thomas Laurence Richie,

Thomas L. Richie, Consulting, USA

Reviewed by:

Laurent Renia,

Agency for Science, Research and

Technology, Singapore Else Bijker,

Radboud University Medical Center, Netherlands

*Correspondence: Patrick E. Duffy, Laboratory of Malaria Immunology and Vaccinology, National Institute of Allergy and Infectious Diseases,

National Institutes of Health,

5640 Fishers Lane, Room-1111,

Rockville, MD 20852, USA

duffype@niaid.nih.gov:

Tejram Sahu,

Laboratory of Malaria Immunology and Vaccinology, National Institute of

Allergy and Infectious Diseases,

National Institutes of Health,

12735 Twinbrook Parkway,

Room-3W15, Rockville, MD 20852,

USA

sahut@mail.nih.gov

Specialty section:

This article was submitted to Microbial Immunology, a section of the journal Frontiers in Microbiology

Received: 20 December 2014

Paper pending published:

07 February 2015

Accepted: 22 March 2015

Published: 09 April 2015

Citation:

Sahu T, Lambert L, Herrod J, Conteh S, Orr-Gonzalez S, Carter D and Duffy PE (2015) Chloroquine neither eliminates liver stage parasites nor delays their development in a murine Chemoprophylaxis Vaccination model.

Front. Microbiol. 6:283. doi: 10.3389/fmicb.2015.00283

\section{Chloroquine neither eliminates liver stage parasites nor delays their development in a murine Chemoprophylaxis Vaccination model}

\author{
Tejram Sahu*, Lynn Lambert, Jessica Herrod, Solomon Conteh, Sachy Orr-Gonzalez, \\ Dariyen Carter and Patrick E. Duffy*
}

\begin{abstract}
Laboratory of Malaria Immunology and Vaccinology, National Institute of Allergy and Infectious Diseases, National Institutes
\end{abstract} of Health, Rockville, MD, USA

Chemoprophylaxis Vaccination (CVac) confers long lasting sterile protection against homologous parasite strains in humans, and involves inoculation of infectious sporozoites (SPZ) under drug cover. CVac using the drug chloroquine (CQ) induces preerythrocytic immunity in humans that includes antibody to SPZ and T-cell responses to liver stage (LS) parasites. The mechanism by which CVac with $\mathrm{CQ}$ induces strong protective immunity is not understood as untreated infections do not confer protection. CQ kills blood stage parasites, but its effect on LS parasites is poorly studied. Here we hypothesized that CQ may prolong or perturb LS development of Plasmodium, as a potential explanation for enhanced pre-erythrocytic immune responses. Balb/c mice with or without CQ prophylaxis were infected with sporozoite forms of a luciferase-expressing rodent parasite, Plasmodium yoelii-Luc (Py-Luc). Mice that received primaquine, a drug that kills LS parasites, served as a positive control of drug effect. Parasite burden in liver was measured both by bioluminescence and by qRT-PCR quantification of parasite transcript. Time to appearance of parasites in the blood was monitored by microscopic analysis of Giemsa-stained thick and thin blood smears. The parasite load in livers of CQ-treated and untreated mice did not significantly differ at any of the time points studied. Parasites appeared in the blood smears of both CQ-treated and untreated mice 3 days after infection. Taken together, our findings confirm that CQ neither eliminates LS parasites nor delays their development. Further investigations into the mechanism of $C Q$-induced protection after $C V a c$ are required, and may give insights relevant to drug and vaccine development.

Keywords: Plasmodium, chloroquine, liver-stage, CVac, prepatent

\section{Introduction}

The most successful vaccination approach against malaria involves immunization with whole organism, generally by the liver-infective form called sporozoites (SPZ). SPZ attenuated by radiation (RAS; Nussenzweig et al., 1967; Clyde et al., 1973) or by genetic alterations (GAP; 
Mikolajczak et al., 2010; Vaughan et al., 2010) infect hepatocytes but arrest during liver stage (LS) development and do not emerge in the blood stream. More recently, experimental infection and drug treatment has achieved impressive protection: in humans, inoculation of SPZ under drug cover, referred to here as Chemoprophylaxis Vaccination (CVac), required much lower SPZ doses than RAS to induce sterile immunity that persisted for $>2$ years in four of six individuals (Belnoue et al., 2004; Roestenberg et al., 2011). Chloroquine (CQ) prophylaxis has been studied most extensively as drug cover during $\mathrm{CVac}$, and this regimen generates preerythrocytic immunity that includes anti-sporozoite antibody (Behet et al., 2014) and LS-specific T-cells in animals and humans (Belnoue et al., 2004; Renia, 2008; Bijker et al., 2013).

Chloroquine kills blood stage parasites as they reach the trophozoite stage of development (Yayon et al., 1983), but the effect of CQ on LS parasites has received less attention. It is widely believed that CQ does not affect LS development, allowing the immune system to encounter the full repertoire of LS antigens. However, the more durable protection achieved with CVac after $\sim 16$-fold fewer mosquito bites than required for RAS suggests that drug treatment effects might contribute independently to protection.

The effect of CQ on Plasmodium LS development is not completely settled. Based on an in vivo infection model, Belnoue et al. (2004) reported that CQ does not kill Plasmodium LS at $42 \mathrm{~h}$ post infection with SPZ. However, the effect of CQ on later time points including blood stage emergence has not been studied. Using an in vitro infection model, Fisk et al. (1989) reported a partial schizonticidal effect of CQ on late LS, but the CQ dose was relatively high, and had cytotoxic effects on hepatocytes as well. Here we report a systematic evaluation of the effects of therapeutic concentrations of CQ on late LS development, using a luciferase expressing $P$. yoelii parasite in $\mathrm{Balb} / \mathrm{c}$ mice.

\section{Materials and Methods}

\section{Mice}

Female Balb/c mice, 4-6 week old were purchased from Taconic. Mice were housed in the NIH animal facility under pathogen free conditions and fed with autoclaved food ad libitum. All experiments were performed once mice reached 16 to 18 weeks of age. All experiments were approved and performed as per the guidelines by the Animal Care and Use Committee (ACUC) of NIAID/NIH.

\section{Parasite and Mosquitoes}

Plasmodium yoelii parasites (strain 17XNL), including the parental line and a transgenic line expressing firefly-luciferase and GFP (Plasmodium yoelii-Luc, Py-Luc), were maintained by cycling between Balb/c mice and Anopheles stephensi mosquitoes. Salivary gland SPZs were harvested on days 1418 as described earlier (Ozaki et al., 1984; Guebre-Xabier et al., 1999).

\section{Infection and Evaluation of LS Development by Bioluminescence Imaging}

Female Balb/c mice (16-18 weeks old) were infected with 15,000 freshly dissected SPZ of $P y$-Luc in $100 \mu \mathrm{l}$ of $1 \mathrm{X}$ PBS+2\% normal mouse serum by IV injection. Development of LS was monitored by bioluminescence imaging (BLI) at different time points post-infection as described earlier (Mwakingwe et al., 2009; Miller et al., 2013). Briefly, mice were injected intradermally with $150 \mu \mathrm{l}$ of Rediject D-Luciferin (Caliper Life Sciences, USA), then anesthetized with isoflurane-anesthesia system 8-10 min later to allow measurement of bioluminescence and image acquisition using IVIS-100 animal imager (Caliper Life Sciences, USA). Bioluminescence images were acquired at the following settings: $15 \mathrm{~cm}$ Field of View (FOV), medium binning, and exposure time of 30-60 s. Quantification of luminescence was performed using the ROI settings of the Living Image 4.4 software (Perkin Elmer, USA). ROI were drawn around the abdominal area locating the liver. ROI measurements were expressed as total flux photons per second $(\mathrm{p} / \mathrm{s})$.

\section{Drug Treatment and LS Burden Estimation by Intravital Imaging}

Infected mice comprised three groups that received different treatments in $100 \mu \mathrm{l}$ of PBS by intraperitoneal (IP) route: one group received $0.8 \mathrm{mg} / \mathrm{mouse}$ of $\mathrm{CQ}$ diphosphate (SPZ+CQ; Sigma; Belnoue et al., 2004): one group received $1.5 \mathrm{mg} / \mathrm{mouse}$ of PQ bisphosphate (SPZ+PQ; Sigma; Putrianti et al., 2009); one group received PBS without drug (SPZ). Two drug control groups were also included, whereby mice received CQ or PQ but no SPZ infection. An additional control group received no drug and no SPZ. Drug was delivered at the time of infection (0 hours post infection, hpi) and at 24 hpi. Bioluminescence images were acquired in vivo with IVIS-100 (Perkin Elmer) at different time points after drug injection indicated in the figure legends. BLI was performed on whole livers that had been perfused (10 $\mathrm{ml}$ of RNAse free $1 \mathrm{x}$ PBS) and isolated at sequential time points from 40 hpi onward. Liver samples were also snap-frozen in liquid nitrogen for qRT-PCR.

\section{Determination of LS Burden by qRT-PCR}

Total RNA was extracted from the whole liver as described earlier (Schussek et al., 2013) using RNeasy mini kit (Qiagen Inc). cDNA was synthesized using High-Capacity cDNA Reverse Transcription Kit (Applied Biosystem, Foster City, CA, USA). Gene expression was measured with 1:40 dilutions of cDNA. Standard curve quantitative RT-PCR was performed (Bruna-Romero et al., 2001) in a $20 \mu l$ volume, which includes $1 \mathrm{X}$ ABI Power SYBR master mix (Applied Biosystems) and $0.25 \mu \mathrm{M}$ of either P. yoelii $18 \mathrm{~S}$ rRNA primer (forward- GGGGATTGGTTTTGACGTTTT, reverse- AAGCATTAAATAAAGCGAATA) or mouse $\beta$-actin primers (Forward- GGCTGTATTCCCCTCCAT; reverseCCAGTTGGTAACAATGCAAT). PCR reactions were run on ABI 7500 machine (Applied Biosystems), using the following conditions: $50^{\circ} \mathrm{C}$ for $2 \mathrm{~min} ; 95^{\circ} \mathrm{C}$ for $10 \mathrm{~min}$; 40 cycles of $95^{\circ} \mathrm{C}$ for $15 \mathrm{~s}$ alternating with $60^{\circ} \mathrm{C}$ for $1 \mathrm{~min}$. 
cDNA standards for both $18 \mathrm{~S}$ rRNA and $\beta$-actin were prepared as 10 -fold dilutions $\left(10^{7}-10^{3}\right.$ copies $)$ from purified PCR product. Liver of naïve mouse was used as negative control. Parasite load was normalized to host $\beta$-actin as a ratio (absolute copy of $P y$ 18S/ absolute copy of mouse $\beta$-actin).

\section{Determination of Prepatent Period}

Thick and thin blood smears were collected from the infected mice at different time points starting $42 \mathrm{hpi}$. Blood smears were Giemsa-stained and examined with a bright field microscope with $100 \times$ oil-immersion objective and by expert slide readers blinded to the study groups. Blood smears were considered positive if at least two infected RBCs were found in 100 adjacent fields.

\section{Statistical Analysis}

Mann-Whitney test was used to compare groups for LS burden measured by either BLI or qPCR. $P \leq 0.05$ was considered statistically significant. GraphPad Prism software (version 6) was used for statistical analysis.

\section{Results}

\section{LS Parasites Persist $54 \mathrm{~h}$ after SPZ Inoculation into Untreated Mice}

Plasmodium undergoes extensive multiplication during LS development, producing tens of thousands of merozoites from an individual sporozoite. Upon completion of LS development, merozoites are released into the blood stream as small merozoitefilled vesicles called merosomes. To quantify the multiplication and subsequent release of parasites in the liver, we infected mice with $1.5 \times 10^{4}$ luciferase expressing $P y$-Luc SPZ and performed BLI at 40,44, 48, 54, and 62 hpi. Parasite biomass increased with time until $44 \mathrm{~h}$ and then gradually declined with the lowest LS parasite burden at 54 hpi (Figures 1 and 2; Supplementary Figure S2). We could detect measurable luciferase activity in the liver at $54 \mathrm{hpi}$ with an average total flux of $5.0 \times 10^{6}$ photon/s (Figure 1). By $62 \mathrm{hpi}$, parasites are

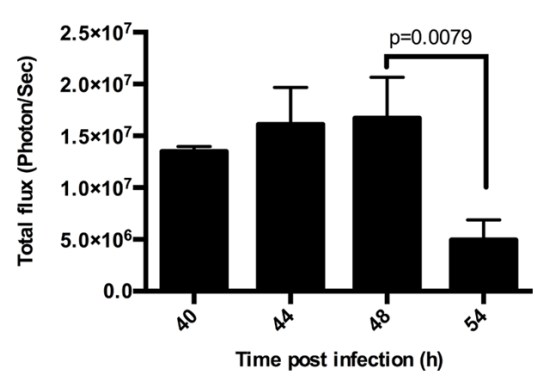

FIGURE 1 | Duration of Plasmodium yoelii-Luc (Py-Luc) liver stage (LS) growth. Graph representing quantification of total flux from infected and untreated mice imaged at 40, 44, 48, and $54 \mathrm{~h}$ after sporozoites (SPZ) injection. $n=3$ for 40 h, 4 for 44 h, and 5 each for 48, and 54 h. Graph represents Mean $\pm \mathrm{SD}$. Mann-Whitney test was performed and $p<0.05$ considered as significant. detected throughout the body, indicating a blood stage infection (Supplementary Figure S1E), and any residual parasites in the liver could not be distinguished from circulating blood stage parasites.

\section{CQ Neither Kills LS Parasites Nor Delays Their Development}

To evaluate the effect of CQ on developing LS parasites, we infected mice with $1.5 \times 10^{4} P y$-Luc SPZ, and then treated with either CQ (0.8 $\mathrm{mg} /$ mouse), PQ (1.5 mg/mouse), or an equal volume of PBS. Three groups of uninfected mice received same treatments (CQ, PQ, or PBS) but no SPZ. Imaging of whole bodies or of isolated livers was performed at 40,44, 48, and 54 hpi. CQ-treated (SPZ+CQ) and untreated (SPZ) mice did not differ significantly at any time point (Figures 2 and 3; Supplementary Figure S2), whereas the SPZ+PQ group was completely devoid of any bioluminescence, as expected (Figure 2; Supplementary Figure S1). Parasite burden estimation with conventional qPCR gave similar results (Figure 2D). At $40 \mathrm{hpi}$, bioluminescence appeared lower in SPZ+CQ group than SPZ group but this trend was not statistically significant $(p=0.06$; Figure 2C), and no such trend was observed in the corresponding qRT-PCR measurements at this time point $(P=0.86$; Figure 2D).

To remove inter-subject variability in a second experiment, we followed individual mice over time, and acquired BLI at sequential time points. We infected each of 20 mice with SPZ of $P y$-Luc; half were treated with CQ while half received an equal volume of PBS. At 42, 44, 46, and 48 hpi, LS parasite burden was measured by whole body BLI. We did not observe significant differences in the parasite burden in the SPZ+CQ versus SPZ animals at any time point (Supplementary Figures S3 and S4). In a third experiment, using parental Py-17XNL infection, we confirmed by qPCR that the LS parasite burden at 48 hpi does not significantly differ between SPZ+CQ and SPZ animals $(p=0.38$, Supplementary Figure S5). Both SPZ+CQ and SPZ mice became blood stage positive at 48 hpi by blood smear microscopy, further indicating that CQ does not delay LS development (Figure 4).

\section{Discussion}

The effect of CQ on Plasmodium blood stages is well characterized, with the inhibition of heme-polymerase enzyme leading to accumulation of toxic heme products, and parasite death (Slater and Cerami, 1992). CQ-CVac generates strong LS-specific immune responses and more durable protection at a log-fold lower SPZ dose as compared to RAS immunization (Clyde et al., 1973; Bijker et al., 2013). However, it is not clear how CQ treatment helps generate this strong and durable immune response. One school of thought holds that CQ-CVac allows the complete development of LS parasites that express a wide array of LS specific antigens, prior to killing the parasites in their first cycle of blood stage development; however, untreated Plasmodium infections also display the full antigen repertoire without inducing sterile preerythrocytic immunity. We hypothesized that 
A

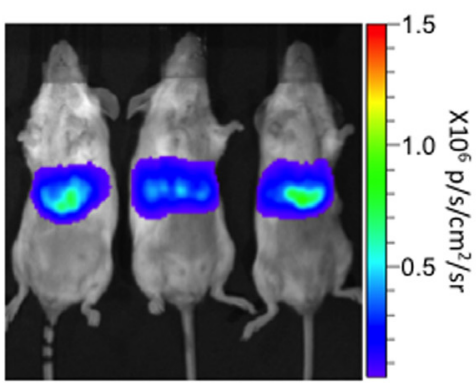

C

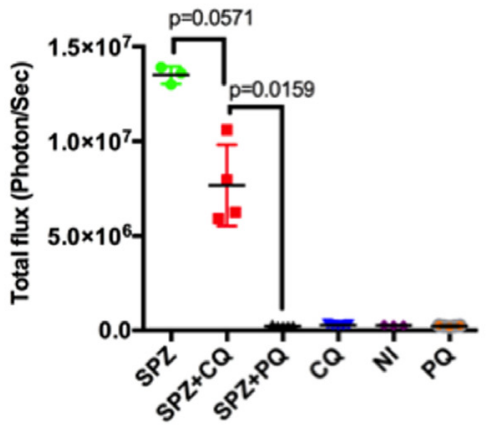

B

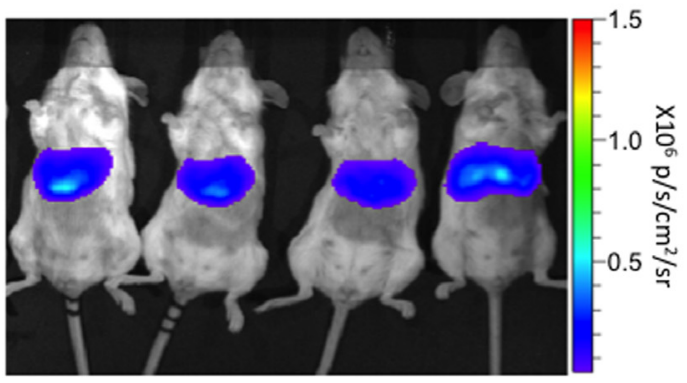

D

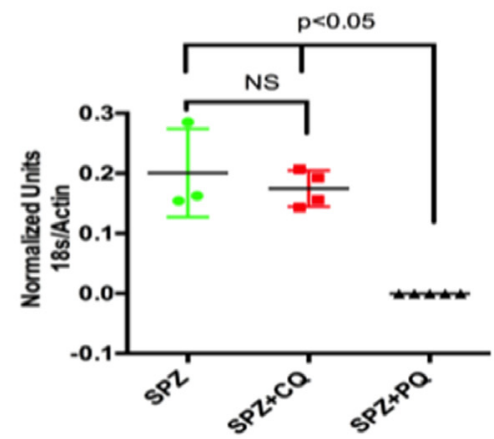

FIGURE 2 | Effect of CQ on LS parasite at $\mathbf{4 0}$ hours post infection (hpi). Rainbow images of mice infected and untreated (A) or CQ-treated (B) showing parasite load in liver $40 \mathrm{~h}$ after injection of $1.5 \times 10^{4} \mathrm{Py}$-Luc salivary gland SPZ. Rainbow scale represents radiance $\left(\mathrm{p} / \mathrm{s} / \mathrm{cm}^{2} / \mathrm{sr}\right)$. (C) Quantification of total flux from whole body imaging of mice (shown in $\mathbf{A}$ and Supplementary Figure S1). $n=3$ for SPZ ( infected and untreated) and $\mathrm{NI}$ (neither infected nor treated) group, four for SPZ+CQ group and five each for SPZ+PQ and drug control (treated uninfected) groups. (D) Quantification of LS parasite burden 40 hpi in livers of SPZ $(n=3)$, SPZ+CQ $(n=4)$, and SPZ+PQ $(n=5)$ mice by qPCR. Graph represents Py-18S RNA normalized to murine $\beta$-actin RNA. Data in $\mathbf{( C , D )}$ represents Mean $\pm \mathrm{SD}$, Mann-Whitney test was performed and $p<0.05$ considered as significant. $\mathrm{CQ}$, Chloroquine; $\mathrm{PQ}$, Primaquine; NI, non-infected and non-treated.

\section{A}

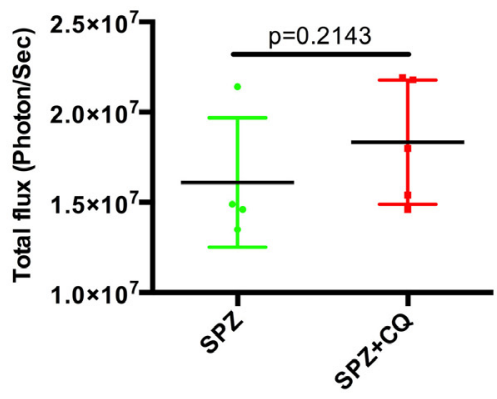

B

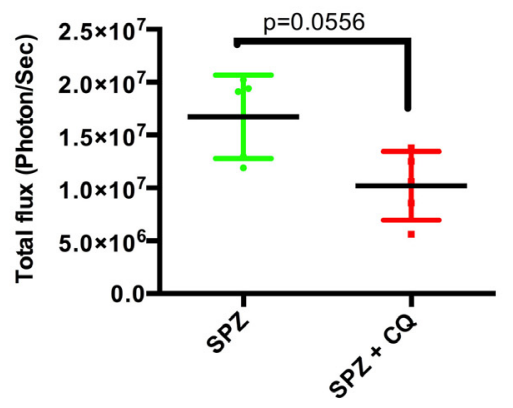

C

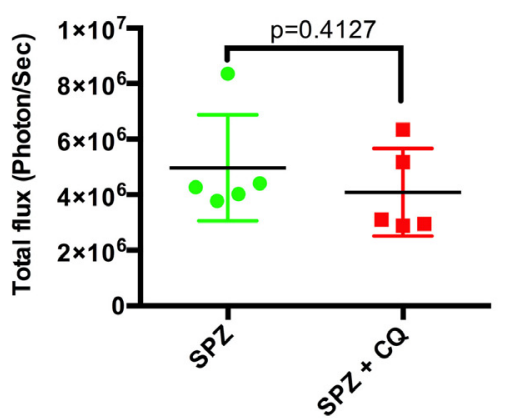

FIGURE 3 | Parasite load in liver at different time points after CQ treatment. Quantification of total flux from whole body imaging of mice (shown in Supplementary Figure S4). $n=4$ for SPZ group at 44 hpi (A), five each for both SPZ and SPZ+CQ at 48 h (B), and 54 h (C) time points. Graph represents mean \pm SD. Mann-Whitney test was performed and $p<0.05$ considered as significant.

CQ may have partial or subtle effects on late LS development that contribute to enhanced immune responses, but we find no evidence that CQ decreases LS burden or delays LS development.

The effect of CQ on the LS of Plasmodium has previously been studied in vivo in a mouse model (Belnoue et al., 2004) and in vitro by infection of non-human primate hepatocytes (Fisk et al., 1989). Belnoue et al. (2004) reported that mice treated with $0.8 \mathrm{mg}$ CQ and untreated mice had similar LS parasite burden $42 \mathrm{hpi}$, but did not examine effects beyond this time point; the CQ dose ( $32 \mathrm{mg} / \mathrm{kg}$ ) used by Belnoue et al. (2004, and also by us for this study) was based on earlier work (Orjih et al., 1982), and is in the dosing range (30-35 mg/kg) used for children of 10-20 kg body weight (Moore et al., 2011). 


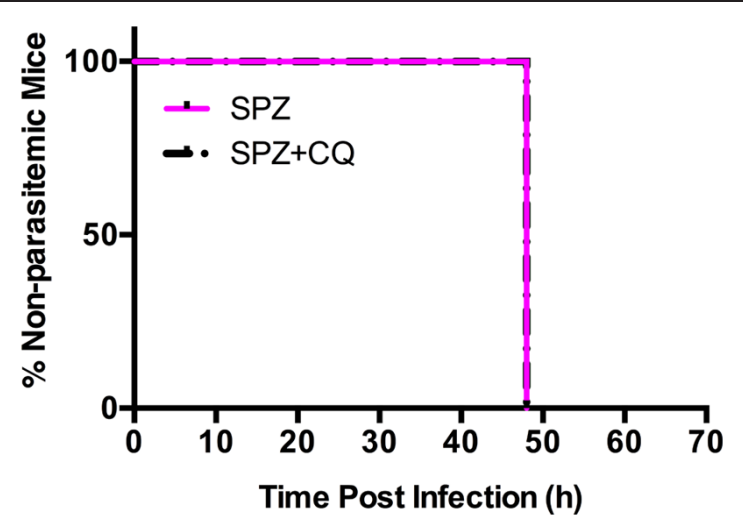

FIGURE 4 | Effect of CQ on prepatent period of $\boldsymbol{P} \boldsymbol{y}$-Luc. Mice $(n=18)$ were infected with $1.5 \times 10^{4} P y$-Luc salivary gland SPZ and either $\mathrm{CQ}$-treated (SPZ+CQ; $n=9$ ) or not (SPZ; $n=9$ ). Blood smears (both thick and thin smears) were collected starting from 48 hpi. Smears were stained and examined by expert smear reader blinded. Smears are considered positive if minimum two parasites were found in 100 adjacent fields.

Earlier, Fisk et al. (1989) reported that CQ at $1 \mu \mathrm{g} / \mathrm{ml}$ had a partial parasiticidal effect in vitro on late LS $P$. cynomolgi and P. knowlesi schizonts. However, these drug concentrations were $\sim 2.5$-fold higher than therapeutic concentrations (0.03-0.4 $\mu \mathrm{g} / \mathrm{ml})$ in humans (Tett et al., 1989; Carmichael et al., 2003), and had toxic effects on hepatocytes that may have contributed to parasiticidal activity.

We examined the effect of CQ on LS development in vivo by imaging luciferase expressing $P$. yoelii parasites in Balb/c mice, and followed parasite development in the liver, both by BLI and qPCR, at multiple time points from $40 \mathrm{~h}$ through $62 \mathrm{~h}$. We observed no difference in the LS parasite load between CQtreated $(\mathrm{SPZ}+\mathrm{CQ})$ and untreated (SPZ) groups at any time point during late LS development. Our observations at 42 hpi are consistent with those of Belnoue et al. (2004) and additionally showed no differences at $44,46,48$, and $54 \mathrm{hpi}$.

We hypothesized that CQ might delay LS development, at least for a subset of parasites, thereby creating a condition of prolonged antigen exposure that might enhance LS specific immunity. All mice, both SPZ+CQ and SPZ, had parasites detectable by blood smear microscopy by 48 hpi, suggesting that there had been no delay in LS development with CQ treatment. This was further substantiated by similar LS parasite burdens in both groups of mice at 48 hpi (Figure 3; Supplementary Figures S3-S5). Our study cannot exclude the possibility that a small subset of parasite persist in liver of SPZ+CQ mice, because emerging blood stage parasites might mask the presence of LS forms in bioluminescence or qRT-PCR studies.

The potential effect of CQ to delay the prepatent period has also been examined in humans as part of CVac trials, using highly sensitive qPCR techniques to detect, and quantify blood stage parasites at densities below the sensitivity of microscopy (i.e, subpatent parasitemia; Roestenberg et al., 2009; Bijker et al., 2013). During CVac, blood stage parasites are detected by qPCR at the time expected of untreated individuals (Roestenberg et al., 2009; Bijker et al., 2013), although a direct comparison of blood stage parasite burden between CQ-treated and untreated individuals has not been reported. Similarly, we find that blood stage parasites are detected by microscopy at $48 \mathrm{hpi}$ in both SPZ $+C Q$ and SPZ mice. A previous study in mice reported that CQ suppressed blood stage growth during CVac (Peng et al., 2014), as is observed in human studies (Roestenberg et al., 2009; Bijker et al., 2013). Our results in mice confirm that CQ does not delay LS development nor kill LS parasites, and support the notion that the diminished first wave of parasitemia is likely to be solely due to its effects on blood stage growth, rather than the effect on the number of parasites released from liver.

Chloroquine was once widely used as an anti-malarial drug and was highly effective in clearing blood stage forms of sensitive Plasmodium parasites, but use of CQ has waned decisively with the global spread of CQ-resistant parasites. Recently, the impressive immunity observed after CVac has sparked renewed interest in CQ as a component of whole organism vaccines, whereby individuals are inoculated with infectious SPZ under CQ drug cover. However, the mechanisms by which drug treatment converts infectious SPZ into an effective vaccine remain unclear. Using a luciferase-expressing parasite and an in vivo mouse infection model, we have systematically evaluated the effect of CQ on Plasmodium late liver stages. We report that CQ given at a dose used for CVac studies neither kills nor delays LS parasite development. However, our study does not exclude other effects of CQ on Plasmodium LS biology that might impact immune responses, such as an altered repertoire of expressed antigens, and hence further investigation is warranted.

\section{Author Contributions}

Conceived and designed the experiments: TS and PD. Performed the experiments: TS, LL, JH, SC, SO-G, and DC. Analyzed the data: TS and PD. Wrote the manuscript: TS and PD.

\section{Acknowledgments}

We thank Prof. Jean Langhorne for her comments and suggestions on this work. We thank Dr. Stephan H. Kappe from Seattle Biomedical Research Institute, Seattle for providing the $P y$-Luc parasite line. Technical help by Yvette Robbins during experiments and Dr. Ankur Sharma during manuscript preparation is duly acknowledged. This work was funded by the Intramural Research Program, NIAID, NIH.

\section{Supplementary Material}

The Supplementary Material for this article can be found online at: http://www.frontiersin.org/journal/10.3389/fmicb.2015.0028 3/abstract 


\section{References}

Behet, M. C., Foquet, L., Van Gemert, G. J., Bijker, E. M., Meuleman, P., LerouxRoels, G., et al. (2014). Sporozoite immunization of human volunteers under chemoprophylaxis induces functional antibodies against pre-erythrocytic stages of Plasmodium falciparum. Malar. J. 13:136. doi: 10.1186/1475-2875-13-136

Belnoue, E., Costa, F. T., Frankenberg, T., Vigario, A. M., Voza, T., Leroy, N., et al. (2004). Protective T cell immunity against malaria liver stage after vaccination with live sporozoites under chloroquine treatment. J. Immunol. 172, 2487-2495. doi: 10.4049/jimmunol.172.4.2487

Bijker, E. M., Bastiaens, G. J., Teirlinck, A. C., Van Gemert, G. J., Graumans, W., Van De Vegte-Bolmer, M., et al. (2013). Protection against malaria after immunization by chloroquine prophylaxis and sporozoites is mediated by preerythrocytic immunity. Proc. Natl. Acad. Sci. U.S.A. 110, 7862-7867. doi: $10.1073 /$ pnas. 1220360110

Bruna-Romero, O., Hafalla, J. C., Gonzalez-Aseguinolaza, G., Sano, G., Tsuji, M., and Zavala, F. (2001). Detection of malaria liver-stages in mice infected through the bite of a single Anopheles mosquito using a highly sensitive real-time PCR. Int. J. Parasitol. 31, 1499-1502. doi: 10.1016/S0020-7519(01)00265-X

Carmichael, S. J., Charles, B., and Tett, S. E. (2003). Population pharmacokinetics of hydroxychloroquine in patients with rheumatoid arthritis. Ther. Drug Monit. 25, 671-681. doi: 10.1097/00007691-200312000-00005

Clyde, D. F., Most, H., Mccarthy, V. C., and Vanderberg, J. P. (1973). Immunization of man against sporozite-induced falciparum malaria. Am. J. Med. Sci. 266, 169-177. doi: 10.1097/00000441-197309000-00002

Fisk, T. L., Millet, P., Collins, W. E., and Nguyen-Dinh, P. (1989). In vitro activity of antimalarial compounds on the exoerythrocytic stages of Plasmodium cynomolgi and P. knowlesi. Am. J. Trop. Med. Hyg. 40, 235-239.

Guebre-Xabier, M., Schwenk, R., and Krzych, U. (1999). Memory phenotype CD8(+) $\mathrm{T}$ cells persist in livers of mice protected against malaria by immunization with attenuated Plasmodium berghei sporozoites. Eur. J. Immunol. 29, 3978-3986.

Mikolajczak, S. A., Vaughan, A. M., Soliman, J. M., and Kappe, S. H. (2010). A genetically attenuated parasite vaccine does not require liver stage persistence to elicit sterile protective immunity against sporozoite-induced malaria in mice. J. Infect. Dis. 201, 1270-1271. doi: 10.1086/651279

Miller, J. L., Murray, S., Vaughan, A. M., Harupa, A., Sack, B., Baldwin, M., et al. (2013). Quantitative bioluminescent imaging of pre-erythrocytic malaria parasite infection using luciferase-expressing Plasmodium yoelii. PLoS ONE 8:e60820. doi: 10.1371/journal.pone.0060820

Moore, B. R., Page-Sharp, M., Stoney, J. R., Ilett, K. F., Jago, J. D., and Batty, K. T. (2011). Pharmacokinetics, pharmacodynamics, and allometric scaling of chloroquine in a murine malaria model. Antimicrob. Agents Chemother. 55, 3899-3907. doi: 10.1128/AAC.00067-11

Mwakingwe, A., Ting, L. M., Hochman, S., Chen, J., Sinnis, P., and Kim, K. (2009). Noninvasive real-time monitoring of liver-stage development of bioluminescent Plasmodium parasites. J. Infect. Dis. 200, 1470-1478. doi: 10.1086/606115

Nussenzweig, R. S., Vanderberg, J., Most, H., and Orton, C. (1967). "Protective immunity produced by the injection of $\mathrm{x}$-irradiated sporozoites of Plasmodium berghei." Nature 216, 160-162. doi: 10.1038/216160a0

Orjih, A. U., Cochrane, A. H., and Nussenzweig, R. S. (1982). Comparative studies on the immunogenicity of infective and attenuated sporozoites of Plasmodium berghei. Trans. R. Soc. Trop. Med. Hyg. 76, 57-61. doi: 10.1016/00359203(82)90019-0

Ozaki, L. S., Gwadz, R. W., and Godson, G. N. (1984). Simple centrifugation method for rapid separation of sporozoites from mosquitoes. J. Parasitol. 70, 831-833. doi: $10.2307 / 3281779$

Peng, X., Keitany, G. J., Vignali, M., Chen, L., Gibson, C., Choi, K., et al. (2014). Artesunate versus chloroquine infection-treatment-vaccination defines stagespecific immune responses associated with prolonged sterile protection against both pre-erythrocytic and erythrocytic Plasmodium yoelii infection. J. Immunol. 193, 1268-1277. doi: 10.4049/jimmunol.1400296

Putrianti, E. D., Silvie, O., Kordes, M., Borrmann, S., and Matuschewski, K. (2009). Vaccine-like immunity against malaria by repeated causal-prophylactic treatment of liver-stage Plasmodium parasites. J. Infect. Dis. 199, 899-903. doi: $10.1086 / 597121$

Renia, L. (2008). Protective immunity against malaria liver stage after vaccination with live parasites. Parasite 15, 379-383. doi: 10.1051/parasite/2008153379

Roestenberg, M., Mccall, M., Hopman, J., Wiersma, J., Luty, A. J., Van Gemert, G. J., et al. (2009). Protection against a malaria challenge by sporozoite inoculation. N. Engl. J. Med. 361, 468-477. doi: 10.1056/NEJMoa0805832

Roestenberg, M., Teirlinck, A. C., Mccall, M. B., Teelen, K., Makamdop, K. N., Wiersma, J., et al. (2011). Long-term protection against malaria after experimental sporozoite inoculation: an open-label follow-up study. Lancet 377, 1770-1776. doi: 10.1016/S0140-67361160360-7

Schussek, S., Groves, P. L., Apte, S. H., and Doolan, D. L. (2013). Highly sensitive quantitative real-time PCR for the detection of Plasmodium liver-stage parasite burden following low-dose sporozoite challenge. PLOS ONE 8:e77811. doi: 10.1371/journal.pone.0077811

Slater, A. F., and Cerami, A. (1992). Inhibition by chloroquine of a novel haem polymerase enzyme activity in malaria trophozoites. Nature 355, 167-169. doi: $10.1038 / 355167 \mathrm{a} 0$

Tett, S. E., Cutler, D. J., Day, R. O., and Brown, K. F. (1989). Bioavailability of hydroxychloroquine tablets in healthy volunteers. Br. J. Clin. Pharmacol. 27, 771-779. doi: 10.1111/j.1365-2125.1989.tb03439.x

Vaughan, A. M., Wang, R., and Kappe, S. H. (2010). Genetically engineered, attenuated whole-cell vaccine approaches for malaria. Hum. Vaccin. 6, 107-113. doi: 10.4161/hv.6.1.9654

Yayon, A., Vande Waa, J. A., Yayon, M., Geary, T. G., and Jensen, J. B. (1983). Stage-dependent effects of chloroquine on Plasmodium falciparum in vitro. J. Protozool. 30, 642-647. doi: 10.1111/j.1550-7408.1983. tb05336.x

Conflict of Interest Statement: The authors declare that the research was conducted in the absence of any commercial or financial relationships that could be construed as a potential conflict of interest.

Copyright (c) 2015 Sahu, Lambert, Herrod, Conteh, Orr-Gonzalez, Carter and Duffy. This is an open-access article distributed under the terms of the Creative Commons Attribution License (CC BY). The use, distribution or reproduction in other forums is permitted, provided the original author(s) or licensor are credited and that the original publication in this journal is cited, in accordance with accepted academic practice. No use, distribution or reproduction is permitted which does not comply with these terms. 